\title{
Time-resolved Optical Studies, Heat Dissipation and Melting of Ag and Au Nanoparticle Systems and Arrays
}

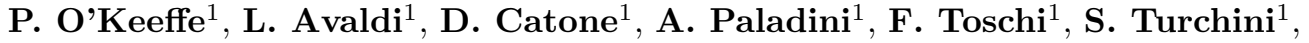 \\ A. Cartoni ${ }^{2}$, I. Fratoddi ${ }^{2}$, and I. Venditti ${ }^{2}$ \\ ${ }^{1}$ CNR-ISM, Rome, Italy \\ ${ }^{2}$ Department of Chemistry, Università "Sapienza", 5 Piazzale Aldo Moro, Rome, Italy
}

\begin{abstract}
Transient absorption spectroscopy has been extensively used in recent years to examine the temporal response of isolated nanoparticles (NPs) to the absorption of light [1]. These studies are largely based on the use of the surface plasmon resonance (SPR) to monitor characteristics of the NP such as electronic and lattice temperature, shape and morphology as a function of time. In the case of extended $\mathrm{Au} / \mathrm{Ag} \mathrm{NP}$ structures the plasmon resonance is strongly distorted due to the inter-particle coupling effects. For example, we have observed this effect in Rhodamine dye functionalized Au nanoparticles which undergo self-assembly to form nanostructures due to the interactions between the dye molecules attached to the surfaces of the nanoparticles. Indeed the SPR splits into two with one resonance remaining in the vicinity of that of the isolated AuNPs and is generally called the transverse SPR while a second resonance due to an extended excitation spanning across multiple particles appears to the lower energies. The precise spectral energy and shape of the extended plasmon resonance depends on the interparticle distance, the particle disposition and the number of particles involved. When the plasmon band or interband spectral region of the NP is excited by an intense pulse the photon energy absorbed by the electrons is transferred to the lattice of the NP as heat through electron-phonon coupling. Depending on the intensity of the light pulse and thus the initial electron temperature a number of outcomes are possible. The first aim of this work is to use low intensity pump pulses to study the wavelength dependence of the sub $10 \mathrm{ps}$ dynamics which reflects the electron-photon scattering within the nanoparticle structure.
\end{abstract}

On the other hand, the interaction of more intense light with the NPs can modify the morphology of NP systems, for example by reshaping gold nanorods into nanospheres or, in general, mediate the synthesis of metallic nanostructures. At medium intensities the initial temperature is sufficient to induce melting of the NPs which can lead to morphological changes of the NP structure. Higher intensities can cause other effects such as photofragmentation of the NPs, release of stabiliser molecules from the surface of the NPs or even Coulomb explosion due to multiple ionisation events. The second aim of this work is to concentrate on the effects of medium intensity laser excitation of a self-assembled $\mathrm{Au} / \mathrm{Ag} \mathrm{NP}$ systems. The NP system is excited by a femtosecond laser pulse of different wavelengths allowing selective deposition of energy and the subsequent heat dissipation through phonon-phonon coupling and morphological changes are monitored in time by recording transient absorption spectra in the visible range. This wavelength range makes it possible to follow the phonon-phonon coupling effects on the recovery of the bleaching of both the transverse and extended plasmon resonances of the NP system. As the intensity of the pump pulse is increased it can be seen that the NPs are no longer able to dissipate all of the heat before arrival of subsequent laser pulses thus leading to melting of the NP structure and strong changes in the plasmon response of the system.

The overall aim of this study is to fully understand the delocalized electron-phonon coupling in the extended plasmon region of the NP structures and to use this knowledge to control the melting in nanostructures. The methods developed can be useful for plasmon mediated nano-engineering and photothermal therapy.

\section{REFERENCES}

1. Hartland, G. V., "Optical studies of dynamics in noble metal nanostructures," Chem. Rev., Vol. 111, No. 6, 3858-3887, 2011. 